\title{
Modification of Expanded Graphite Electrodes by Ozone Treatment
}

\author{
P. KRAWCZYK AND J.M. SKOWROŃSKI* \\ Institute of Chemistry and Technical Electrochemistry, Poznań University of Technology \\ Piotrowo 3, 60-965 Poznań, Poland
}

\begin{abstract}
The aim of the present work was the modification of the electrochemical properties of expanded graphite by ozone treatment. Electrochemical investigations showed that ozone modification of expanded graphite electrodes results in the significant improvement of their electrochemical activity towards the phenol carried out by cyclic voltammetry method in alkaline solution. The highest activity demonstrates sample expanded graphite modified under ozone atmosphere at elevated temperature. The analysis of Fourier transform infrared spectra and voltammetric curves recorded in electrolyte without phenol additive showed increased concentration of different types of oxygen functional groups on the surface of modified expanded graphite.
\end{abstract}

PACS numbers: 82.45.Fk, 82.47.-a, 82.44.Wx, 82.80.Fk

\section{Introduction}

Due to fact that phenol and its derivatives pertain to the most dangerous pollutants, there is a need to remove them from the water solutions. The processes attempting to this purpose are realized by direct oxidation of pollutant at the surface of electrode material [1-3], and/or via indirect methods by the electrochemical formation of oxidative agent, e.g., active oxygen and hydroxyl radicals [4]. Under some conditions the electrochemical methods gives scope for entire oxidation of phenol leading to its transformation into final products $\mathrm{CO}_{2}$ and water. This process is accompanied by the formation of variety of soluble (e.g., hydroquinone, benzoquinone, fumaric and maleic acids) and insoluble (oligomer film) by-products [1-6]. Taking into consideration the efficiency of phenol oxidation, the generation of oligomeric coating the electrode surface should be regarded as an unwanted behavior. The appearance of oligomer makes an electrode almost completely inactive due to the blocking its active centers for phenol molecules. In consequence, the process of phenol oxidation may be inhibited and even entirely interrupted. A variety of attempts were performed to solve the problem of electrode fouling by oligomeric compounds generated during the initial steps of phenol oxidation. One of them concerns the preliminary activation of electrode resulting in enhancement its electrochemical activity and/or increased resistivity against deactivation [5]. The other methods based on the processes of regeneration of electrode materials being exhausted of oligomeric products of phenol oxidation, allow the restoration and even enhancement of the initial

* corresponding author; e-mail: jan.skowronski@put.poznan.pl electrochemical activity of applied electrodes [7-9]. In the latter case, chemical treatment of electrode material with ozone seems to be very useful technique because ozone can play a role of both an activating and regenerating agent. From the literature [10-14] it is known that process of ozonation of carbon materials brings about the increase of their oxidation degree due to a significant enrichment of the carbon surface with oxygen functional. Among the functionalities generated during the ozonation, the carboxylic, carbonyl and lactonic group can be found at the surface of carbon materials [10-13]. The formation of radical compounds within the carbon structure should also be considered as an effect of ozonation $[10,15]$. It cannot be excluded that the ozone treatment of carbon materials, especially proceeding at elevated temperatures, may also contribute to the changes in the crystalline and porous structure of carbons [10,11].

Expanded graphites (EGs) being the products of the process of exfoliation of graphite intercalation compounds (GICs) exhibit interesting physicochemical features, such as enhanced adsorption capacity, developed surface area, resistivity against heating $[16,17]$, which allow their practical application in electrochemical processes not only as an electrode material but also as a catalyst.

The purpose of the present work was to study the influence of ozone modification of EG on its electrochemical properties. The electrochemical properties of ozonated EG were examined by cyclic voltammetry technique in a model reaction of phenol electrooxidation occurring in alkaline solution. The electrochemical properties of ozonated EG were complemented by analysis of its surface chemical composition performed on the basis of the Fourier transform infrared (FTIR) spectra. 


\section{Experimental}

Synthetic graphite powder composed of particles with diameters ranging from 32 to $71 \mu \mathrm{m}$ was used as a host material to obtain graphite intercalation compound with sulfuric acid $\left(\mathrm{H}_{2} \mathrm{SO}_{4}-\mathrm{GIC}\right)$ by the method of anodic oxidation in $18 \mathrm{M} \mathrm{H}_{2} \mathrm{SO}_{4}$ [18]. $\mathrm{H}_{2} \mathrm{SO}_{4}$-GIC thus prepared was thermally exfoliated in a muffle furnace at the temperature of $800^{\circ} \mathrm{C}$ in air for $4 \mathrm{~min}$ to obtain expanded graphite.

The process of ozone modification was conducted in glass reactor under continuous flow of ozone through the bed of expanded graphite. Ozone was produced from the air supplying the ozone generator with the flow rate of $0.12 \mathrm{~m}^{3} / \mathrm{h}$. The process was carried out at room temperature and at $160^{\circ} \mathrm{C}$. During the reaction time $1 \mathrm{~h}$, the amount of $206.7 \mathrm{mg}$ ozone was passed through the reactor. The stability of thermal conditions upon modification was ensured by the use of oil bath.

The cyclic voltammetry measurements with a scan rate $0.1 \mathrm{mV} / \mathrm{s}$ were performed in the potential range from the rest potential of electrode $\left(E_{\mathrm{R}}\right)$ to $0.8 \mathrm{~V}$ using $0.1 \mathrm{M}$ phenol in $0.5 \mathrm{M}$ aqueous solution of $\mathrm{KOH}$. The potentials on all presented voltammograms are related to the $\mathrm{Hg} / \mathrm{HgO} / 0.5 \mathrm{M} \mathrm{KOH}$ reference electrode. To examine the chemical nature of EG electrodes, the electrochemical measurements in electrolyte without phenol admixture were performed. In this experiment scan range was adjusted to $-0.95 \leftrightarrow 0.8 \mathrm{~V}$, starting from the $E_{\mathrm{R}}$ towards the less positive potentials. In each case the mass of working electrode was equal to $30 \mathrm{mg}$. The powder type working electrodes were prepared according to the procedure described in detail in previous papers $[5,8,9]$. Expanded graphite was placed in a pocket made of porous polymer material, in which graphite rod (5 $\mathrm{mm}$ in diameter), playing a role of current collector, was beforehand inserted. A similar graphite rod was used as a counter electrode. The reference electrode was connected to the solution under investigation by a Luggin capillary. Before starting measurements working electrode was equilibrated at an opened circuit for $1 \mathrm{~h}$. Electrochemical measurements were performed using AUTOLAB potentiostat-galvanostat (model PGSTAT 30). Phenol used as reagent was purchased from LACHEMA (puriss > 99.5 wt\%).

FTIR measurements were performed with BRUKER-model $113 \mathrm{~V}$ IR spectrometer using the $\mathrm{KBr}$ technique.

\section{Discussion}

Figure 1 displays cyclic voltammograms (CVs) recorded in $0.1 \mathrm{M}$ phenol in $0.5 \mathrm{M}$ solution of $\mathrm{KOH}$ for electrode made of the original EG. The huge anodic peak recorded for the first cycle at the potential of $0.43 \mathrm{~V}$ has been previously ascribed to the reaction of phenol electrooxidation $[5,8,9,19,20]$. During the subsequent cycle, the current charges as well as the intensity of the anodic peak rapidly fall down due to the blocking of active

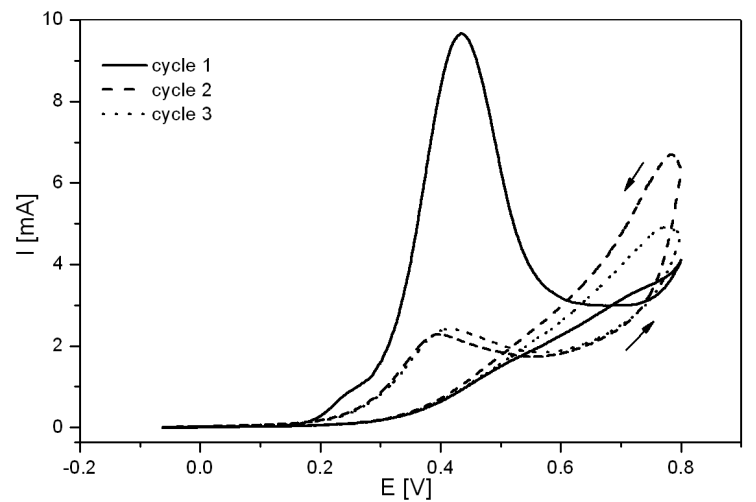

Fig. 1. Cyclic voltammograms recorded for the original EG in $0.1 \mathrm{M}$ phenol in $0.5 \mathrm{M} \mathrm{KOH}$. Scan range $E_{\mathrm{R}} \leftrightarrow 0.8 \mathrm{~V}$; scan rate $0.1 \mathrm{mV} / \mathrm{s}$.

surface of EG by oligomeric products generated during the precedent phenol oxidation.

The current charge related to the regarded peak decreases from $12.01 \mathrm{C}$ for the first cycle to $1.94 \mathrm{C}$ for the second cycle. The peak charges for all the investigated samples are given in Table. Since the peak charge calculated for the third cycle is very close to that for the second cycle it allows the supposition that the downward tendency does not develop. Such a behaviour suggests that the process of phenol oxidation upon cycling proceeds at the surface of oligomeric film instead of the surface of EG. Anodic peaks positioned at the potential of $0.78 \mathrm{~V}$ for the second and third cycle can be tentatively ascribed to the secondary reactions of phenol oxidation or/and the oxidation reactions involving by-products previously formed.

TABLE

Peak charges calculated for all investigated samples.

\begin{tabular}{c|c|c|c}
\hline \hline Sample & $\begin{array}{c}\text { Cycle 1 } \\
{[\mathrm{C}]}\end{array}$ & $\begin{array}{c}\text { Cycle 2 } \\
{[\mathrm{C}]}\end{array}$ & $\begin{array}{c}\text { Cycle 3 } \\
{[\mathrm{C}]}\end{array}$ \\
\hline EG & 12.01 & 1.94 & 2.13 \\
EG-o-p & 17.20 & 4.84 & 2.53 \\
EG-o-t & 20.76 & 9.75 & 7.95
\end{tabular}

Because the process of phenol electrooxidation is almost entirely interrupted due to irreversible deactivation of EG electrode by the oligomeric products, therefore there is the necessity to find the effective method of EG modification ensuring its increased activity as well as enhanced resistivity against fouling.

In Fig. 2 there are shown voltammograms recorded during electrochemical oxidation of $0.1 \mathrm{M}$ phenol at electrode made of ozone modified EG. The subjected modification was made by passing the gaseous ozone through the bed of EG beforehand placed in a glass reactor. The reaction between ozone and EG was carried out for one hour at ambient temperature. 


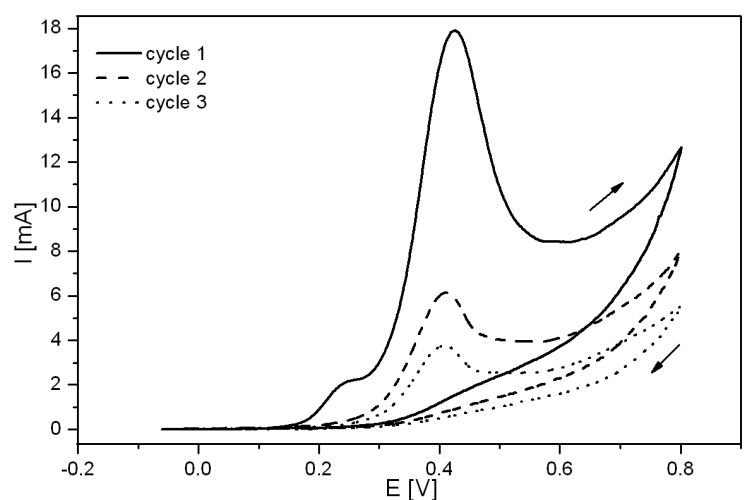

Fig. 2. Cyclic voltammograms for EG after its ozone modification at room temperature (EG-o-p) recorded in $0.1 \mathrm{M}$ phenol in $0.5 \mathrm{M} \mathrm{KOH}$. Scan range $E_{\mathrm{R}} \leftrightarrow 0.8 \mathrm{~V}$; scan rate $0.1 \mathrm{mV} / \mathrm{s}$.

The sample of EG after mentioned modification was denoted as EG-o-p. Taking into consideration the charge of anodic peak associated with phenol oxidation as the main criterion of the estimation of electrochemical activity, it can be pointed out that ozone treatment of EG resulted in significant increase of its activity towards the process of phenol electrooxidation. $T$ current charge of anodic peak for the first cycle is over $40 \%$ higher as compared to the untreated EG (Table). Such a behaviour may indicate that ozone treatment of EG favours the formation of the oxygen surface functional groups and, in consequence gives rise to the enhancement of its electrochemical activity towards the process of phenol oxidation. It should be emphasized that in contrast to the original EG, the activity of EG resulted from its ozone treatment remains on the enhanced level also during the subsequent cycle of oxidation. The peak charge noted for the second cycle for the regarded sample is over twice higher than that for the original EG (Table). It is likely that the changes in chemical structure of the EG surface affected by ozone treatment have also influence on the features of the oligomeric film generated during the phenol oxidation on the surface of modified sample. The above hypothesis is supported by the increased activity demonstrated by EG during the second cycle as well as the differences in $\mathrm{CV}$ characteristics observed during the further anodic oxidation. As can be seen in Fig. 2, there is no current loop, arising from secondary reactions of phenol oxidation similar to those previously observed for untreated sample within the potential range $0.6 \div 0.8 \mathrm{~V}$ (Fig. 1).

To examine the influence of the temperature of EG ozone modification on its electrochemical activity, the following modification was carried out at elevated temperature.

Figure 3 shows cyclic voltammograms recorded during phenol oxidation at EG electrode modified by ozone treatment performed for $1 \mathrm{~h}$ at the temperature of $160^{\circ} \mathrm{C}$ (the sample denoted EG-o-t).

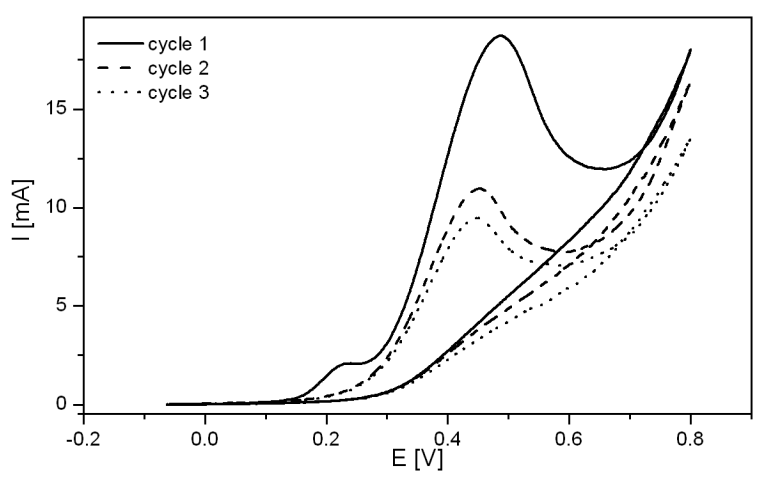

Fig. 3. Cyclic voltammograms for EG after its ozone modification at temperature of $160{ }^{\circ} \mathrm{C}$ (EG-o-t) recorded in $0.1 \mathrm{M}$ phenol in $0.5 \mathrm{M} \mathrm{KOH}$. Scan range $E_{\mathrm{R}} \leftrightarrow 0.8 \mathrm{~V}$; scan rate $0.1 \mathrm{mV} / \mathrm{s}$.

The comparison of CVs shown in Figs. 1, 2 and 3 clearly indicates that ozonation performed at elevated temperature markedly improves the electrochemical properties of EG being demonstrated by the increase of its electrochemical activity as well as the changes in the mechanism and kinetics of phenol electrooxidation. For the first cycle, the current charge of anodic peak ascribed to phenol oxidation of over $72 \%$ higher than that noted for the original sample EG and of over $20 \%$ higher as compared to the charge calculated for the sample modified at ambient temperature (Table). It is reasonable to infer that despite the diminution of electrochemical activity after the first oxidation cycle, the activity level of sample EG-o-t upon cycling is much higher than that observed for both unmodified EG and EG treated with ozone at room temperature. Such a behaviour probably indicates that the unwanted electrode deactivation affected by covering the EG surface by oligomeric products is significantly inhibited due to ozone modification carried out at temperature of $160^{\circ} \mathrm{C}$. As it was previously mentioned, the increase of temperature of ozonation also contributes to the alteration of the mechanism and kinetics of considered process. As can be seen in Fig. 3 the anodic peak related to phenol oxidation becomes wider along with the rapid increase of current observed in the potential region of $0.65 \div 0.8 \mathrm{~V}$. Moreover, the maxima of anodic peaks corresponding to phenol oxidation are shifted by about $60 \mathrm{mV}$ towards the more positive potentials (Fig. 3) as compared to samples EG (Fig. 1) and EG-o-p (Fig. 2).

Summarizing these results, one can assume that the surface of modified EG is considerably enriched in oxygen functional groups and other active agents such as oxygen radicals playing a role of active centres during the process of phenol electrooxidation. It is known from the literature that the ozone treatment of different types of carbon materials may result in the generation of several types of active complexes [10-13, 15] and even the structural changes, if ozonation is carried out at elevated temperature $[10,11]$. 
To investigate the particular influence of ozone treatment on the modification of chemical composition of EG surface, the electrochemical measurements in electrolyte free of phenol were performed. Figures 4,5 and 6 display $\mathrm{CVs}$ recorded in $0.5 \mathrm{M} \mathrm{KOH}$ in the potential range $-0.95 \leftrightarrow 0.8 \mathrm{~V}$ for samples EG, EG-o-p and EG-o-t, respectively. To reveal the effects arising from the reaction of functional groups originally present on the surface of EG electrode, the measurements were started from the rest potential of electrode $\left(E_{\mathrm{R}}\right)$ towards the less positive potentials. On all the CVs recorded instantly after the beginning of the measurement, the cathodic current effects, corresponding to the reduction reactions of the surface functional groups, appear. Making an assumption that the intensity of the regarded cathodic peaks are in coincidence with the concentration of surface functionalities undergoing electrochemical reduction, it is possible to estimate the creative role of ozone modification of EG. Based on the above supposition it can be stated that functional complexes of highest concentration appear on the surface of EG modified by ozone at elevated temperature (EG-o-t) (Fig. 6).

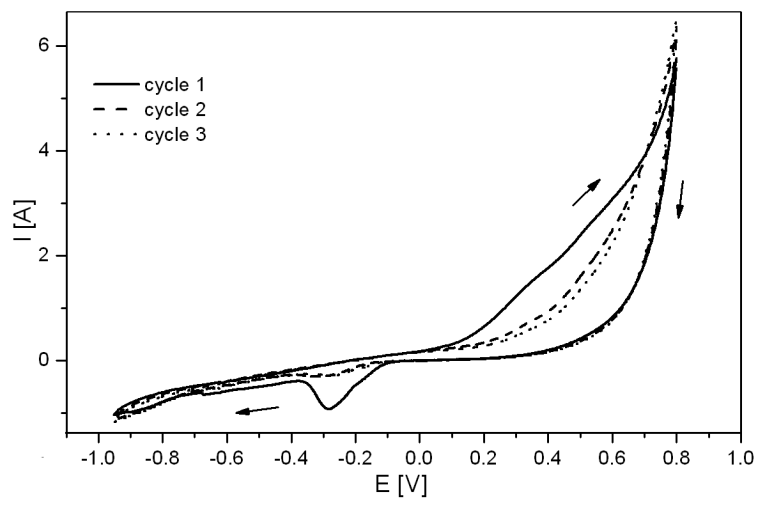

Fig. 4. Cyclic voltammograms for the original EG recorded in $0.5 \mathrm{M} \mathrm{KOH}$. Scan range $-0.95 \leftrightarrow 0.8 \mathrm{~V}$; scan rate $0.1 \mathrm{mV} / \mathrm{s}$.

For sample EG-o-t a broad cathodic peak of the highest intensity with the maximum is observed at around $-0.50 \mathrm{~V}$. This peak is preceded by a small cathodic peak at $-0.23 \mathrm{~V}$. Peak spreading in a wide potential range illustrates the reduction reactions of various functional groups differing between each other in the bonding energy. In contrast, markedly less amount of surface functionalities was estimated for the original EG (Fig. 4). In this case the cathodic part of $\mathrm{CV}$ curves includes a distinct peak positioned at $-0.28 \mathrm{~V}$.

Apart from the cathodic reactions, the modification has also a strong influence on the anodic characteristics of EG. As can be seen in Figs. 5 and 6, when the electrodes reach the potential of $0.65 \mathrm{~V}$ the current rapidly grows up, due to starting the reaction of oxygen evolution. The oxidation reaction is responded during the cathodic polarisation in the second and third cycle. Instead of small peak located at the potential of $-0.23 \mathrm{~V}$,

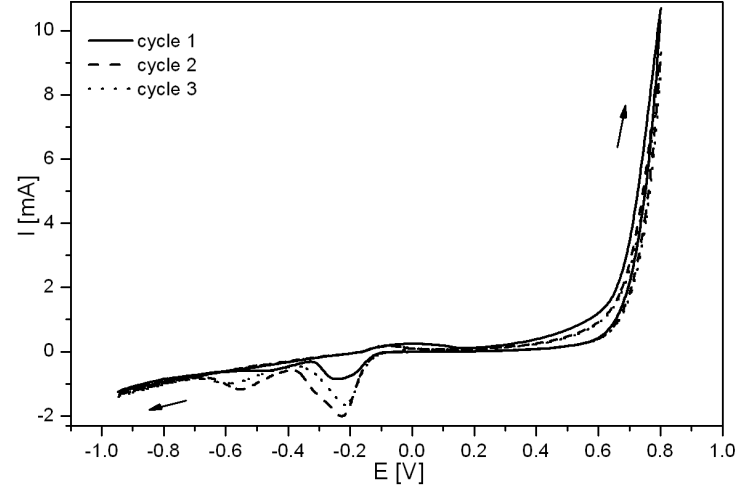

Fig. 5. Cyclic voltammograms for EG after ozone modification at room temperature (EG-o-p) recorded in $0.5 \mathrm{M} \mathrm{KOH}$. Scan range $-0.95 \leftrightarrow 0.8 \mathrm{~V}$; scan rate $0.1 \mathrm{mV} / \mathrm{s}$.

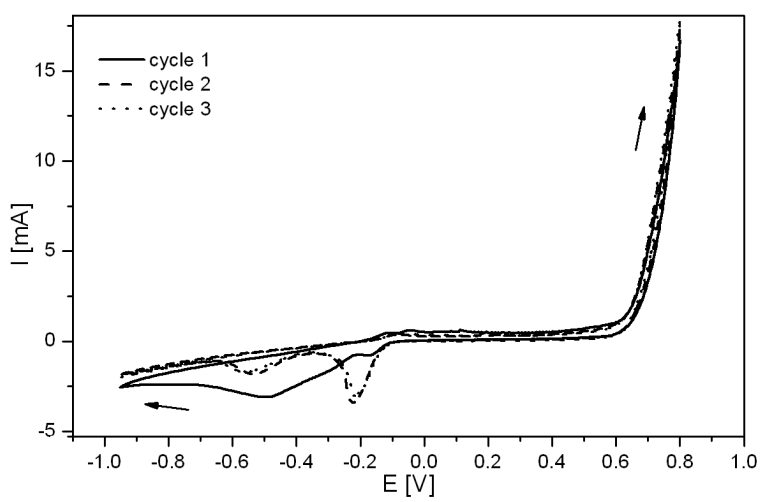

Fig. 6. Cyclic voltammograms for EG after ozone modification at temperature of $160{ }^{\circ} \mathrm{C}$ (EG-o-t) recorded in $0.5 \mathrm{M} \mathrm{KOH}$. Scan range $-0.95 \leftrightarrow 0.8 \mathrm{~V}$; scan rate $0.1 \mathrm{mV} / \mathrm{s}$.

enlarged peak appears at the potential slightly shifted towards the negative potentials. On the other hand, a broad cathodic peak diminishes considerably and attains its maximum at the potential of $-0.53 \mathrm{~V}$. The peaks can be considered in term of reduction reactions of functionalities formed at the surfaces of EG due to the reactions of oxygen evolution. It is likely that similar oxidation reactions occur simultaneously with the process of phenol oxidation realized at electrodes made of modified EG. If so, the processes related to oxygen sorption and evolution resulting the formation of functional groups may be responsible for the enhanced activity of EG-o-t due to increased concentration of active sites on its surface during the successive cycles of oxidation (Fig. 3).

Much more valuable information in term of functional groups generated on the ozone modified surface of EG are given from the FTIR analysis. Figure 7 shows FTIR spectra recorded for all the investigated samples.

From the literature it is known that ozone treatment of carbons brings about the formation of various types of 


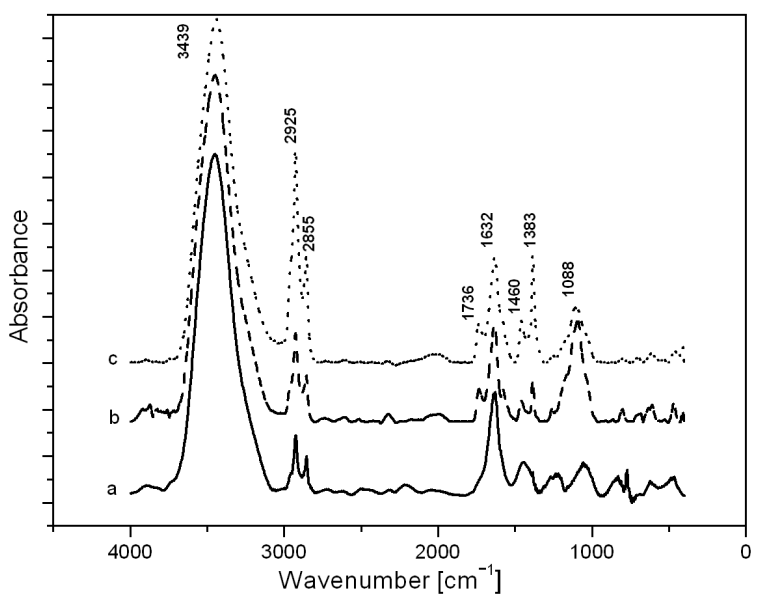

Fig. 7. FTIR spectra for: $a$ - original EG, $b$ - sample EG after its ozone modification at room temperature (EG-o-p), $c$ - sample EG after its ozone modification at temperature of $160{ }^{\circ} \mathrm{C}$ (EG-o-t).

oxygen functional groups [10-15] as well as characteristic bonds containing $\mathrm{C}$ and $\mathrm{H}$ atoms [10]. Generally, among the oxygen functionalities created by ozonation of active carbons, the carboxylic, ether, alcoholic, carbonyl, lactone and ester groups are considered [10-15]. The above mentioned information is in coincidence with that arising from the analysis of FTIR spectra recorded for EG after its ozone modification (Fig. 7). The comparison of all the FTIR spectra reveals that in case of modified EG samples (b and c) some new bands appear. The most important from the viewpoint of electrochemical activity seems to be the bands at 1088, 1383 and $1736 \mathrm{~cm}^{-1}$, probably corresponding to: (i) $\mathrm{C}-\mathrm{O}$ bonds in ether, alcoholic groups [13, 21]; (ii) $\mathrm{C}-\mathrm{O}$ pertaining to carboxylic groups $[10,11,22]$ and (iii) $\mathrm{C}=\mathrm{O}$ attributed to carboxylic, lactone and carbonyl groups [13]. On all the FTIR spectra shown in Fig. 7, one can find other bands derived from the structure of expanded graphite. Two of them, positioned at 2855 and $2925 \mathrm{~cm}^{-1}$ arising from stretching vibrations of $\mathrm{C}-\mathrm{H}$ bonds $[10,22]$ seem to be worth to note since their intensity is strongly influenced by the type of the investigated sample. Considerably enlarged effects were attained for sample EG-o-t (spectrum c), whereas the lowest for the original EG (spectrum a). Such a relation gives evidence that, during the modification, the formation of compounds containing $\mathrm{C}-\mathrm{H}$ bond may occur in parallel with oxygen functionalities. Chiang et al. [10] have found that such bonds are created within the structure of active carbons during its ozone activation.

From the comparison of spectra $\mathrm{a}$ and $\mathrm{b}$ one can infer that the most pronounced effects of the process of ozone modification at $160{ }^{\circ} \mathrm{C}$ are observed in quantitative changes occurring in chemical composition of the EG surface. Among them, the change in the intensity of signal at $1383 \mathrm{~cm}^{-1}$ seems to be the most valuable. For sample EG-o-t (spectrum c) the regarded signal is over twice higher as compared to that of sample EG-o-p (spectrum b), suggesting that the higher concentration of $\mathrm{C}-\mathrm{O}$ bond pertaining to carboxylic groups appears on the surface of EG modified in ozone atmosphere at elevated temperature. Based on this statement, it is reasonable to assume that the increased amount of carboxyl functional group existing on the surface of sample EG-o-t is responsible for its enhanced activity in the process of phenol oxidation.

\section{Conclusion}

Electrochemical investigations showed that ozone modification of EG electrodes results in the significant improvement of their electrochemical activity towards the process of phenol oxidation occurring in alkaline solution. Taking into account the value of current charge as a main criterion of electrochemical activity assessment, it can be pointed out that the highest activity was found for sample EG modified in ozone atmosphere at the temperature of $160^{\circ} \mathrm{C}$. The peak charge for this sample calculated for the first cycle over $72 \%$ exceeds the charge for unmodified sample EG. In spite of the fact that electrochemical activity falls down after the first oxidation cycle it still remains on a higher level than those observed for both the original EG and EG treated in ozone at room temperature. The electrochemical measurements in electrolyte free of phenol, confirmed by the FTIR analysis, indicate that functional groups formed on the surface of EG during ozone oxidation may be responsible for its enhanced activity towards the phenol oxidation. The particular analysis of FTIR spectra showed the increased concentration of carboxylic group on the surface of EG after its modification carried out at elevated temperature. It gives evidence that the appearance of such groups makes this sample to be much more electrochemically active as compared to the sample modified with ozone at room temperature.

\section{Acknowledgments}

This work was financially supported from the grant of the Poznań University of Technology No. DS 31-151/08.

\section{References}

[1] M. Gottrell, D.W. Kirk, J. Electrochem. Soc. 139, 2736 (1992).

[2] S. Andreescu, D. Andreescu, O.A. Sadik, Electrochem. Commun. 5, 681 (2003).

[3] M.S. Ureta-Zañartu, P. Bustos, C. Berrios, M.C. Diez, M.L. Mora, C. Gutiérrez, Electrochim. Acta 47, 2399 (2002).

[4] H. Kuramitz, Y. Nakata, M. Kawasaki, S. Tanaka, Chemosphere 45, 37 (2001).

[5] P. Krawczyk, J.M. Skowroński, Przem. Chem. 8-9, 1198 (2006).

[6] J.M. Skowroński, P. Krawczyk, J. Solid State Electrochem. 11, 223 (2007). 
[7] F. Salvador, C. Sánchez, Jiménez, Carbon 34, 511 (1996).

[8] J.M. Skowroński, P. Krawczyk, J. Solid State Electrochem. 8, 242 (2004).

[9] J.M. Skowroński, P. Krawczyk, Chem. Eng. J. 152, 464 (2009).

[10] H.-L. Chiang, C.P. Huang, P.C. Chiang, Chemosphere 47, 257 (2002).

[11] H.-L. Chiang, P.C. Chiang, C.P. Huang, Chemosphere 47, 267 (2002).

[12] H. Valdés, M. Sánchez-Polo, J. Rivera-Utrilla, C.A. Zaror, Langmuir 18, 2111 (2002).

[13] P.M. Álvarez, J.F. García-Araya, F.J. Beltrán, F.J. Masa, F. Medina, J. Colloid Interface Sci. $\mathbf{2 8 3}$ $503(2005)$.

[14] Z. Jin, Z. Zhang, L. Meng, Mater. Chem. Phys. 97, 167 (2006).
[15] H. Valdés, C.A. Zaror, J. Hazard. Mater. B 137, 1042 (2006).

[16] D.D.L. Chung, J. Mater. Sci. 22, 4190 (1987).

[17] G. Furdin, Fuel 77, 479 (1998).

[18] J.M. Skowroński, K. Jurewicz, Synth. Met. 40, 161 (1991).

[19] J.M. Skowroński, P. Krawczyk, in: 51th Annual Meeting of Int. Soc. of Electrochemistry, Warszawa, Extended Abstracts 2000.

[20] J.M. Skowroński, P. Krawczyk, in: Int. Carbon Symp. EUROCARBON, Oviedo, Extended Abstracts 2003.

[21] V. Gómez-Serrano, P.M. Álvarez, J. Jaramillo, F.J. Beltrán, Carbon 40, 523 (2002).

[22] S. Biniak, G. Szymański, J. Siedlewski, A. Swiątkowski, Carbon 35, 1799 (1997). 